\title{
Heat Transfer in Laminar Flow Through a Tube ${ }^{1}$
}

\author{
Milton Abramowitz, ${ }^{2}$ William F. Cahill, ${ }^{3}$ and Clarence Wade, $\mathrm{Jr}^{3}$
}

\begin{abstract}
The problem of heat transfer due to laminar flow of a viscous fluid in a channel is studied under the assumption that there is a parabolic distribution of velocity. The effect of axial temperature changes are considered and the solution is based on the simpler situation where axial effects are discussed. The solution, obtained by the method of least squares, is represented in terms of a set of nonorthogonal characteristic functions. These functions and the corresponding characteristic values are determined by numerical integration employing the Runge-Kutta procedure. Finally, asymptotic developments are obtained which are useful in the limiting cases.
\end{abstract}

\section{Statement of Problem}

The present paper is concerned with the steady state problem of heat transfer in a tube. The fluid moves with a prescribed velocity profile, which is parabolic in the present case. Thus the end effect is not considered in the velocity profile. The heat transfer is small and is supposed not to influence the fluid motion. The wall of the tube is assumed to be kept at a fixed temperature, $\theta_{1}$, while the temperature of the fluid entering the tube is fixed to be $\theta_{0}$. The velocity distribution in the tube whose radius is unity is defined by

$$
v_{x}=2 v_{m}\left(1-r^{2}\right), 0 \leq r \leq 1 \text {, }
$$

where $r$ is the radial distance from the center and $v_{x}$ is taken along the $x$-axis which is the axis of symmetry of the tube and $v_{m}$ is the average value of $v_{x}$. The equation of heat transfer under these conditions is

$$
v_{x} \frac{\partial \theta}{\partial x}=K\left\{\frac{\partial^{2} \theta}{\partial r^{2}}+\frac{1}{r} \frac{\partial \theta}{\partial r}+\frac{\partial^{2} \theta}{\partial x^{2}}\right\}
$$

with the boundary conditions

$$
\begin{gathered}
\theta=\theta_{0} \text { for } x=0 \\
\theta \text { finite for } x=\infty \\
\theta=\theta_{1} \text { for } r=1 .
\end{gathered}
$$

This problem has been considered $[1]^{4}$ under the

\footnotetext{
1 The preparation of this paper was sponsored in part by the Office of Naval Research, United States Navy.

2 Deceased.

${ }_{3}^{3}$ Present address, Theoretical Division, National Aeronautics and Space Administration.

4 Figures in brackets indicate the literature references at the end of this paper.
}

assumption that temperature changes in the $x$ direction will be negligible so that the term $\partial^{2} \theta / \partial x^{2}$ may be omitted. However, recent studies [2,3], which have included the effects of this term have exhibited solutions based on approximating the temperature distribution by a series of Bessel functions. In the present paper we propose to obtain a solution by the method of least squares. The boundary value problem will be solved by numerical integration of the differential equation employing the Runge-Kutta method. This procedure avoids the use of special functions but employs the basic concepts necessary to solve the boundary value problem. The results obtained indicate that the present technique may be employed in other problems of a similar nature.

Let us first put $\lambda=2 v_{m} / K$ and $\lambda \xi=x$ so that (1.2) becomes

$$
\left(1-r^{2}\right) \frac{\partial \theta}{\partial \xi}=\frac{\partial^{2} \theta}{\partial r^{2}}+\frac{1}{r} \frac{\partial \theta}{\partial r}+\frac{1}{\lambda^{2}} \frac{\partial^{2} \theta}{\partial \xi^{2}}
$$

while the boundary conditions (1.3) remain unchanged except that $x$ is replaced by $\xi$. We see from (1.4) that when $\lambda$ increases the contribution from the term $\partial^{2} \theta / \partial \xi^{2}$ will decrease. By analogy with the solution for $\lambda=\infty$ it is assumed that

$$
\frac{\theta-\theta_{1}}{\theta_{0}-\theta_{1}}=\sum_{n=1}^{\infty} A_{n} \exp \left(z_{n} \xi\right) y\left(r, z_{n}\right)
$$

where the constants $z_{n}$ are the eigenvalues and the functions $y\left(r, z_{n}\right)$ are the eigenfunctions of the boundary value problem

$$
\begin{gathered}
y^{\prime \prime}+\frac{1}{r} y^{\prime}+\left\{\frac{z_{n}^{2}}{\lambda^{2}}-z_{n}\left(1-r^{2}\right)\right\} y=0, \\
y(r, \lambda, z)=0 \text { for } r=1 .
\end{gathered}
$$


TABLE 1. The eigenvalues- $z_{n}$

\begin{tabular}{|c|c|c|c|c|c|c|}
\hline$n$ & $\lambda^{a}=0$ & $\lambda=1$ & $\lambda=10$ & $\lambda=100$ & $\lambda=100 \mathrm{G}$ & $\lambda=\infty$ \\
\hline 1 & 2. 405 & 2. 644369 & 6. 744051 & 7. 306909 & 7. 313523 & 7. 313587 \\
\hline 2 & 5. 520 & 5. 187345 & 30.76791 & 44. 32333 & 44. 60653 & 44. 66947 \\
\hline 3 & 8.654 & 8. 323452 & 59. 50344 & 111.9926 & 113. 9009 & 113. 9210 \\
\hline 4 & 11. 792 & 11. 46137 & 89. 47665 & 208. 3608 & 215. 1669 & 215. 2447 \\
\hline 5 & 14. 931 & 14. 60050 & 119. 9727 & 330.8685 & 348. 3678 & 348.564 \\
\hline 6 & 18. 071 & 17. 74037 & 150.7501 & 476. 6471 & 513.4475 & 513.890 \\
\hline 7 & 21.212 & 20.88073 & 181. 6979 & 642.7513 & 710. 3849 & 711.217 \\
\hline 8 & 24. 352 & 24. 02119 & 212.7574 & 826. 6381 & 939. 7287 & 940.551 \\
\hline 9 & 27.49 & 27. 16301 & 243. 9199 & 1024.875 & 1196.191 & 1201.87 \\
\hline 10 & 30.63 & 30. 30613 & 275. 1170 & 1224.774 & 1464. 309 & 1495. 20 \\
\hline 11 & 33.78 & 33. 44743 & & & & \\
\hline 12 & 36.92 & 36. 50123 & & & & \\
\hline 13 & 40.06 & 40.06675 & & & & \\
\hline
\end{tabular}

a For $\lambda=0, z_{n}$ are the zeros of the Bessel function $J_{0}(z)$; the values for $\lambda=\infty$ were taken from reference [4].

In order to have axial symmetry it is required that $y(r, \lambda, z)$ be an even function of $r$. Further, to determine the temperature distribution $\theta$ in (1.5), we determine solutions of (1.6) which constitute a nonorthogonal set of characteristic functions $y(r$, $\left.\lambda, z_{n}\right)$. It is noted that since $z$ occurs quadratically in (1.6) there are two sets of characteristic values. It turns out that one set is all positive while the other is all negative. However, in evaluating the temperature distribution, we wish to have a solution which is finite for $\xi$ infinite so we discard the positive set of characteristic values. In order to satisfy the condition $\theta=\theta_{0}$ at $\xi=0$ we formally assume the solution in the form

$$
f(r) \sim \sum_{n=1}^{\infty} A_{n} y\left(r, z_{n}\right)
$$

where $f(r)=1$ in the present problem. When $\lambda=\infty,(1.6)$ reduces to the Graetz-Nusselt equation and the functions $\sqrt{r\left(1-r^{2}\right)}, y\left(r, z_{n}\right)$ constitute an orthogonal set and the coefficients are determined accordingly. In the present case where $\lambda$ is finite the orthogonality property no longer holds. However, we may still determine the coefficients in the sense of least squares. Consequently, we require

$\int_{0}^{1} r\left(1-r^{2}\right)\left\{f(r)-\sum_{n=1}^{\infty} A_{n} y\left(r, z_{n}\right)\right\}^{2} \quad d r=$ minimum.

The weight function $r\left(1-r^{2}\right)$ has been introduced so that the results shall be consistent with the Graetz-Nusselt $(\lambda=\infty)$ case. It should be noted that the solution of (1.6) may be expressed in terms of the confluent hypergeometric function. However, to obtain the required results it would still be necessary to consider the problem of evaluating these functions. Instead, we propose to solve the differential eq (1.6) directly using numerical integration. Differentiating (1.9) with respect to $A_{n}$ we get the infinite system of equations

where

$$
\alpha A=\beta,
$$

$$
\begin{aligned}
& \alpha=\left[\alpha_{m n}\right]=\left[\int_{0}^{1} r\left(1-r^{2}\right) y\left(r, z_{m}\right) y\left(r, z_{n}\right) d r\right], \\
& \beta=\left[\beta_{m}\right]=\left[\int_{0}^{1} r\left(1-r^{2}\right) y\left(r, z_{m}\right) f(r) d r\right] \\
& A=\left[A_{n}\right], m, n=1,2, \ldots .
\end{aligned}
$$

\section{Determination of $z_{n}$ and $y\left(r, z_{n}\right)$}

The solution of the infinite system is dependent on the determination of the characteristic functions $y\left(r, z_{n}\right)$ and the characteristic values $z_{n}$. The power series solution of (1.6) is

$$
y\left(r, z_{n}\right)=\sum_{k=0}^{\infty} b_{2 k} r^{2 k},
$$

where

$$
\begin{aligned}
& b_{0}=1, b_{2}=-\frac{1}{4}\left(\frac{z^{2}}{\lambda^{2}}-z\right), 4 k^{2} b_{2 k}+\left(z^{2} / \lambda^{2}\right. \\
&-z) b_{2 k-2}+z b_{2 k-4}=0 .
\end{aligned}
$$

This representation was used to expedite the numerical integration of (1.6). The Runge-Kutta method [5] was employed starting at $r=0.5$ with the value of $y\left(0.5, z_{n}\right)$ obtained from (2.1) with trial values of $z_{n}$. The results are given in table 1 (see figure 1 for the characteristic functions corresponding to $\lambda=10)$. 


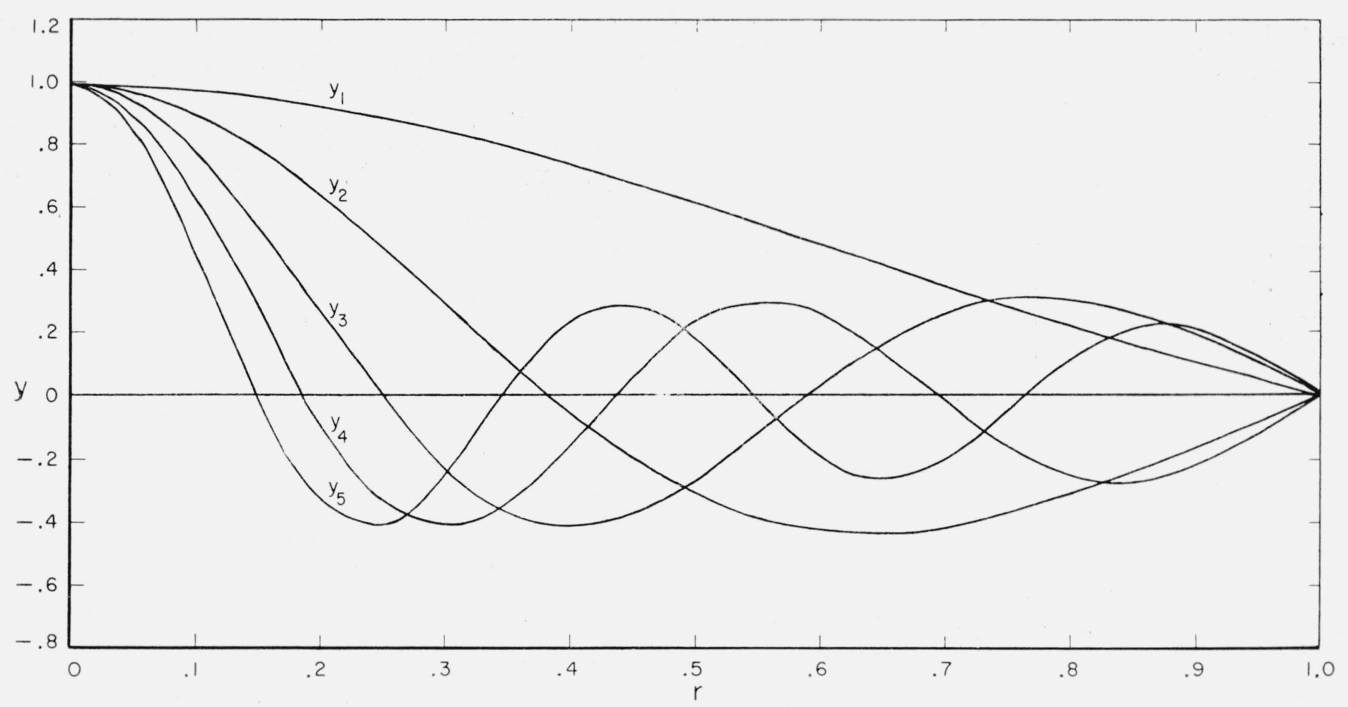

Figure 1.

\section{Calculation of the Coefficients $A_{j}$}

The integrals in (1.11) necessary for the determination of the solution $A$ of (1.10) were evaluated with Simpson's rule using the characteristic functions $y\left(r, z_{n}\right)$ calculated previously. We give the results obtained for 3 cases. Since the matrices are symmetric we omit the elements above the main diagonal.

$10^{8} \alpha=\left(10^{8} \alpha_{m n}\right)=\left[\begin{array}{r}1018886 \\ 1338862 \\ -242794 \\ 77045 \\ -32588\end{array}\right.$

$10^{7} \alpha=\left(10^{7} \alpha_{m n}\right)=\left[\begin{array}{r}947286 \\ 42284 \\ -18966 \\ 8546 \\ -4079\end{array}\right.$

$$
\lambda=1, \quad m=n=5
$$

$\left.\begin{array}{rrrr}3951938 & & & \\ 849201 & 2472921 & 1804961 & - \\ -176352 & 606464 & 469689 & 1422473\end{array}\right]$

$$
\lambda=10, \quad m=n=5
$$
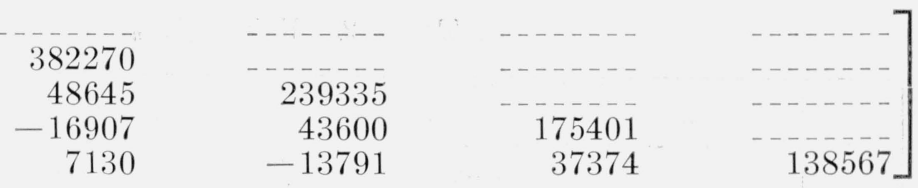

$\lambda=1000, \quad m=n=8$

$$
\begin{array}{r}
939335 \\
5 \\
-12 \\
6 \\
-11 \\
6 \\
8 \\
52
\end{array}
$$
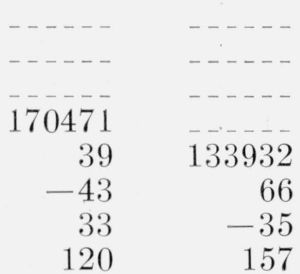
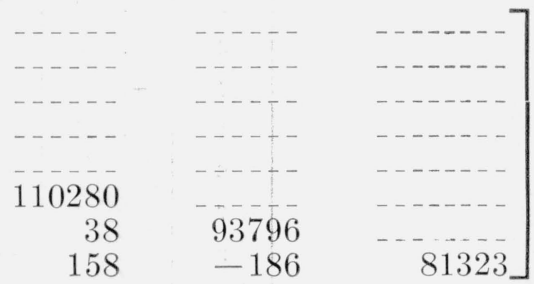
Thus for $\lambda=1$, if we start with the approximations $z_{2}^{(1)}=-5$ and $z_{2}^{(2)}=-6$ to $z_{2}$ the following successive approximations are obtained:

$\begin{array}{lll}j & z_{2}^{(j)} & y\left(1, z_{2}^{(j)}\right) \\ 1 & -6.0 & 0.2362897 \\ 2 & -5.0 & -.6633393 \times 10^{-1} \\ 3 & -5.219196 & .1110549 \times 10^{-1} \\ 4 & -5.187761 & .1455328 \times 10^{-3} \\ 5 & -5.187344 & -.4580215 \times 10^{-6} \\ 6 & -5.187345 & -.2379238 \times 10^{-7}\end{array}$

The improved value of $z_{n}$ was obtained by linear interpolation as follows:

$$
\frac{z_{2}^{(j+1)}-z_{2}^{(j)}}{z_{2}^{(j-1)}-z_{2}^{(j,}}=\frac{y\left(1, z_{2}\right)-y\left(1, z_{2}^{(j)}\right)}{y\left(1, z_{2}^{(j-1)}\right)-y\left(1, z_{2}^{(j)}\right)} ; y\left(1, z_{2}\right)=0 .
$$

The efficiency of the method was dependent on the choice of the initial values of $z$. Once some of the eigenvalues were known, relatively good starting values were easily found by extrapolation. In performing the integration (1.6) was written in the form

$$
\begin{aligned}
& y^{\prime}=\frac{1}{r} u \\
& u^{\prime}=-r\left\{\left(z^{2} / \lambda^{2}-z\right)+z r^{2}\right\} y
\end{aligned}
$$

and the interval $\Delta r=0.002$ was ultimately employed.

It is noted that as $\lambda$ increases, the matrix $\alpha$ tends to a diagonal matrix, i.e., the set of characteristic functions $y\left(r, z_{n}\right)$ tends to an orthogonal set with respect to the weight factor $r\left(1-r^{2}\right)$. Finally, examination of the integrals which are the elements of $\alpha$ and $\beta$ indicates that since $f(r)=1$, elements of $\beta$ follow as a simple byproduct of the computation of the elements of $\alpha$.

Solving the system $\alpha A=\beta$ we get the values of $A_{n}$ in table 2.

With the known values of $A_{n}$ we are able to test the efficiency of the least square approximation. The results are summarized in table 3 .
We conclude from the above that the method described yields satisfactory results for practical purposes. In conclusion we shall give asymptotic representations useful in the regions of small and large values of $\lambda$. These serve to confirm the

\begin{tabular}{|c|c|c|c|c|c|c|c|c|}
\hline \multirow{2}{*}{$r$} & \multicolumn{2}{|c|}{$\lambda=1$} & \multicolumn{2}{|c|}{$\lambda=10$} & \multicolumn{2}{|c|}{$\lambda=100$} & \multicolumn{2}{|c|}{$\lambda=1,000$} \\
\hline & $n=5$ & $n=8$ & $n=5$ & $n=8$ & $n=5$ & $n=8$ & $n=5$ & $n=8$ \\
\hline 0 & 0.981 & 0.918 & 1. 151 & 0.905 & 1. 188 & 0.845 & 1. 188 & 0.864 \\
\hline 0.1 & .937 & 1. 011 & 1. 047 & 1. 009 & 1. 037 & 1. 042 & 1. 033 & 1. 100 \\
\hline .2 & .909 & 1. 004 & 0.935 & 0.997 & 0.921 & 0.972 & 0.922 & 0.961 \\
\hline .3 & 1.004 & 0.985 & 1. 001 & .992 & 1. 042 & 1. 033 & 1.186 & 1.178 \\
\hline .4 & 1.100 & 1. 023 & 1. 057 & 1. 010 & 1. 040 & 0.965 & 1.036 & 0.963 \\
\hline .5 & 1. 053 & 0.971 & 0.969 & 0.972 & 0.920 & 1. 023 & 0.917 & 1.036 \\
\hline .6 & 0.965 & 1. 032 & .942 & 1. 039 & .997 & 0.997 & 1.004 & 0.974 \\
\hline .7 & .990 & 0.969 & 1. 076 & 0.952 & 1. 139 & .964 & 1.142 & .987 \\
\hline .8 & .984 & 1. 024 & 1. 081 & 1. 052 & 0.992 & 1. 121 & 0.983 & 1.126 \\
\hline .9 & .651 & 1. 004 & 0.682 & 0.964 & .539 & 0.758 & .528 & 0.718 \\
\hline 1. 0 & 0 & 0 & 0 & 0 & 0 & 0 & 0 & 0 \\
\hline
\end{tabular}
numerical results obtained previously.

TABLE $3 . \quad \sum_{n=1}^{\infty} A_{n} y\left(r, z_{n}\right) \sim f(r)=1$

\section{Solution of (1.6) for Small Values of $\lambda$}

To obtain a solution of (1.6) in terms of Bessel functions valid for small values of $\lambda$ we put $\alpha \lambda=z$ and obtain

$$
y^{\prime \prime}+r^{-1} y^{\prime}+\left[\alpha^{2}-\alpha \lambda\left(1-r^{2}\right)\right] y=0 .
$$

Now if $\lambda \rightarrow 0,(4.1)$ becomes $y^{\prime \prime}+r^{-1} y^{\prime}+\alpha^{2} y=0$. The solution of the limiting problem is $J_{0}\left(\alpha_{s} r\right)$ where $J_{0}$ is the Bessel function of the first kind of order zero and $\alpha_{s}=j_{s}$ are the zeros of $J_{0}$.

TABLE 2. Values of $A_{n}$

\begin{tabular}{|c|r|r|r|r|r|r|r|r|}
\hline \hline \multirow{2}{*}{$n$} & \multicolumn{2}{|c|}{$\lambda=1$} & \multicolumn{2}{|c|}{$\lambda=10$} & \multicolumn{2}{|c|}{$\lambda=100$} & \multicolumn{2}{|c|}{$\lambda=1,000$} \\
\cline { 2 - 6 } & \multicolumn{1}{|c|}{$n=5$} & $n=8$ & $n=5$ & $n=8$ & $n=5$ & $n=8$ & $n=5$ & $n=8$ \\
\hline 1 & 1.5779 & 1.6003 & 1.5370 & 1.5423 & 1.478413 & 1.479273 & 1.476457 & 1.476472 \\
2 & -0.9629 & 1.0527 & -0.9563 & -0.9778 & -0.8126151 & -0.813778 & -0.806192 & -0.806222 \\
3 & .5217 & 0.8172 & .7559 & .8062 & .599728 & .606701 & .588885 & .588874 \\
4 & -.4547 & -.6658 & -.5894 & -.6865 & -.488371 & -.501968 & -.475988 & -.475937 \\
5 & .2995 & .5489 & .4038 & .5805 & .411076 & .419506 & .405103 & .405024 \\
6 & & -.4462 & & -.4797 & & -.390254 & & -.355635 \\
7 & & .3450 & & .3765 & & .348450 & & .318681 \\
8 & & -.2289 & & -.2565 & & -.303294 & & -.287201 \\
\hline
\end{tabular}


Thus, if we assume

$$
\begin{gathered}
y(r)=y_{0}+\lambda y_{1}+\lambda^{2} y_{2}+\ldots \\
\alpha_{s}(\lambda)=\alpha_{0 s}+\lambda \alpha_{1 s}+\lambda^{2} \alpha_{2 s}+\ldots .
\end{gathered}
$$

and substitute in (4.1) we find

$$
\begin{aligned}
y_{1}(r) & =\left(\frac{1}{6}-\frac{1}{3 j_{s}^{2}}\right) r J_{1}\left(j_{s} r\right)-\frac{1}{2} \frac{r^{2}}{j_{s}^{2}} J_{2}\left(j_{s}^{r}\right)+\frac{r^{3}}{6} J_{3}\left(j_{s}^{r}\right) \\
\alpha_{1 s} & =\frac{1}{3}+\frac{1}{3 j_{s}^{2}}, \alpha_{2 s}=\frac{1}{15 j_{s}}-\frac{7}{90 j_{s}^{3}}+\frac{59}{90 j_{s}^{5}}
\end{aligned}
$$

Omitting further approximations we show the comparison considering the quantities $\alpha_{1 s}$ and $\alpha_{2 s}$.
$\lambda-z_{1}(\lambda)$
Approx. $\quad-z_{2}(\lambda)$
Approx.
$1 \quad 2.04437$
2. 04417
3. 44802
5. 187345
5. 18755
3.48773
6. 0350
9. 75388
9. 7570
5. 68968
15. 2
20. 4943
20. 462
$10 \quad 6.744051$
30. 7679
32.52

\section{Solution of (1.6) for Large Values of $\lambda$}

Let us assume that

$$
\begin{gathered}
y(r)=y_{0}+\lambda^{-2} y_{1}+\lambda^{-4} y_{2}+\ldots \\
z_{s}(\lambda)=-z_{0 s}+z_{1 s} \lambda^{-2}+z_{2 s} \lambda^{-4}+\ldots .
\end{gathered}
$$

We then find that $y_{0}(r)$ is the solution of the GraetzNusselt equation and $z_{0 s}$ are the corresponding eigenvalues which have been listed in table 1. Substituting in (1.6) we obtain a series of characteristic values from which we find

$\begin{array}{lll}s & z_{1 s} & z_{2 s} \\ & -66.9260 & 1218.6 \\ 1 & -2897.8 & 374 \times 10^{3}\end{array}$

A comparison of the results taking into account $z_{1} s$ and $z_{2 s}$ follows:

$\lambda$

$$
-z_{1}(\lambda)
$$$$
-z_{2}(\lambda)
$$

True value Approx. True value Approx.

$\begin{array}{rllll}10 & 6.744 & 6.766 & 30.8 & 53.0 \\ 100 & 7.307 & 7.307 & 44.3 & 44.323 \\ 1000 & 7.3135 & 7.313 & 44.6065 & 44.606 \\ \infty & 7.313587 & & 44.60947 & \end{array}$

The authors acknowledge suggestions and assistance of Miss Irene A. Stegun in the asymptotic developments obtained.

\section{References}

[1] M. Jakob, Heat transfer, vol. 1 (John Wiley \& Sons, Inc., New York, N.Y., 1949), p. 451.

[2] K. Millsaps and K. Pohlhausen, Heat transfer to HagenPoiseuille flows, Proceedings of the Conference on Differential Equations (University of Maryland, College Park, Md., 1955).

[3] S. N. Singh, Heat transfer by laminar flow in cylindrical tube (private communication).

[4] M. Abramowitz, On the solution of the differential equation occurring in the problem of heat convection in laminar flow through a tube, J. Math. Phys. 32, 184 (1953).

[5] William E. Milne, Numerical solution of differential equations, Ch. 5 (John Wiley \& Sons, Inc., New York, N.Y., 1953)

[6] H. A. Lauwerier, The use of confluent hypergeometric functions in mathematical physics and the solution of the eigenvalue problem, Appl. Sci. Research, [A] 2, 184 (1951).

Washington, June 2, 1958. 